\title{
A síndrome da dor patelofemoral altera o movimento do retropé, mas não modifica a distribuição da pressão plantar*
}

\section{Patellofemoral Pain Syndrome Modifies the Movement of the Rearfoot, but It Does Not Alter Plantar Pressure Distribution}

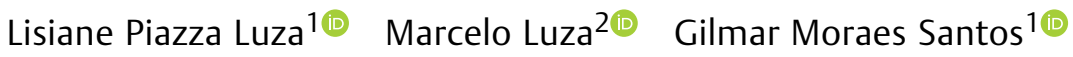 \\ ${ }^{1}$ Laboratório de Postura e Equilíbrio, Centro de Ciências da Saúde e do \\ Esporte, Universidade do Estado de Santa Catarina, Florianópolis, \\ Endereço para correspondência Lisiane Piazza Luza, PhD, \\ SC, Brasil \\ ${ }^{2}$ Universidade de Passo Fundo, Passo Fundo, RS, Brasil \\ Universidade do Estado de Santa Catarina, Rua Pascoal Simone 358, \\ Florianópolis, SC, 88080350, Brasil \\ (e-mail: lisiane_piazza@yahoo.com.br).
}

Rev Bras Ortop 2020;55(4):419-425.

\section{Resumo \\ Palavras-chave \\ - síndrome da dor patelofemoral \\ - fenômenos biomecânicos \\ - joelho \\ - marcha}

Objetivo Comparar a distribuição da pressão plantar e a cinemática do retropé durante a fase de apoio da marcha de sujeitos com e sem síndrome da dor patelofemoral (SDPF).

Métodos Participaram 26 sujeitos com SDPF e 31 clinicamente saudáveis, pareados em idade, estatura e massa corporal. Foi avaliada a distribuição da pressão plantar (pico de pressão) em seis regiões plantares, e a cinemática do retropé (ângulo máximo de eversão do retropé, porcentagem da fase de apoio da marcha em que o ângulo é atingido, e porcentagem da fase de apoio em que o retropé permaneceu em eversão). Os dados foram analisados por meio da estatística descritiva e inferencial, com nível de significância de $p \leq 0,05$.

Resultados A pressão nas seis regiões plantares analisadas e a magnitude do ângulo máximo de eversão do retropé durante a marcha em superfície plana não se mostrou diferente nos sujeitos com SDPF. No entanto, sujeitos com SDPF apresentaram, dentro do ciclo da marcha, ângulo máximo de eversão do retropé mais cedo do que sujeitos do grupo controle, e permaneceram menos tempo com o retropé em eversão.

Conclusão A SDPF parece estar relacionada à alteração no padrão temporal na cinemática do retropé.

\footnotetext{
Abstract

Objective To compare the plantar pressure distribution and the kinematics of the rearfoot on the stance phase of subjects with or without patellofemoral pain syndrome (PFPS).
}

\footnotetext{
Trabalho desenvolvido no Laboratório de Postura e Equilíbrio do Centro de Ciências da Saúde e do Esporte da Universidade do Estado de Santa Catarina (CEFID/UDESC) e no Laboratório de Biomecânica da Universidade de Passo Fundo (UPF).
}

recebido

15 de Janeiro de 2019 aceito

12 de Junho de 2019
DOI https://doi.org/ 10.1055/s-0039-1698802. ISSN 0102-3616.
Copyright $\odot 2020$ by Sociedade Brasileira License terms de Ortopedia e Traumatologia. Published by Thieme Revinter Publicações Ltda, Rio de Janeiro, Brazil 


\section{Keywords \\ - patellofemoral pain syndrome \\ - biomechanical phenomena \\ - knee \\ - gait}

\begin{abstract}
Methods A total of 26 subjects with PFPS and 31 clinically healthy subjects, who were paired regarding age, height and mass, participated in the study. The plantar pressure distribution (peak pressure) was assessed in six plantar regions, as well as the kinematics of the rearfoot (maximum eversion angle, percentage of the stance phase when the maximum angle was reached, and percentage of the stance phase in which the rearfoot was in eversion). The data were analyzed by descriptive and inferential statistics, with a significance level of $p \leq 0.05$.

Results The pressure on the six plantar regions analyzed and the magnitude of the maximum eversion angle of the rearfoot when walking on flat surfaces did not present differences among the subjects with PFPS. However, the PFPS subjects showed, when walking, an earlier maximum eversion angle of the rearfoot than the subjects on the control group, and stayed less time with the rearfoot in eversion.

Conclusion The PFPS seems to be related to modifications on the temporal pattern on the kinematics of the rearfoot.
\end{abstract}

\section{Introdução}

A síndrome da dor patelofemoral (SDPF) caracteriza-se como uma dor difusa na região anterior do joelho, geralmente de início insidioso e progressão lenta, ${ }^{1}$ sendo responsável por $25 \%$ de todas as lesões que acometem essa articulação. ${ }^{2}$ Entre os diversos fatores envolvidos em sua etiologia multifatorial, destacam-se o mau alinhamento patelar, a fraqueza do quadríceps, ${ }^{1}$ as alterações no alinhamento postural dos membros inferiores, especialmente as relativas ao ângulo do retropé, 2,3 além de anormalidades na biomecânica da extremidade inferior.

Barton et al. ${ }^{4}$ afirmam que o conhecimento das diferenças cinemáticas entre os indivíduos com e sem SDPF é importante para os profissionais da saúde e pesquisadores, pois é necessário para desenvolver e otimizar estratégias de prevenção e tratamento para a SDPF. Uma alteração cinemática, como maior eversão do retropé, pode conduzir a uma rotação interna compensatória do fêmur, podendo causar uma maior compressão entre a superfície articular da patela e o côndilo femoral lateral e conduzir aos sintomas patelofemorais. ${ }^{5}$

Adicionalmente, Santos ${ }^{6}$ relata que há uma relação direta positiva entre o ângulo máximo de eversão do retropé e a distribuição da pressão plantar, ou seja, à medida que os valores do ângulo máximo de eversão do retropé aumentam, as pressões plantares nas regiões do médiopé também aumentam. Assim, hipotetizamos que sujeitos com SDPF apresentarão maior ângulo máximo de eversão do retropé durante a marcha, e que esta alteração poderia ocasionar mudanças na distribuição da pressão plantar, com maiores valores para o pico de pressão nas regiões mediais do pé.

Considerando que a distribuição da pressão plantar tem um grande potencial para predizer movimentos anormais durante a locomoção, ${ }^{6}$ torna-se importante avaliar suas características durante a realização das atividades funcionais dos sujeitos com SDPF, pois é durante estas atividades que estes sujeitos mais exacerbam seus sintomas. Além disso, a avaliação da distribuição da pressão plantar poderia acrescentar bases para a reabilitação da SDPF, ajudando a elucidar o comportamento da interface do pé com o solo ou o calçado como um reflexo do alinhamento dinâmico dos membros inferiores. ${ }^{7}$

Segundo Thijs et al., ${ }^{1}$ alterações na distribuição da pressão plantar podem reduzir a função de absorção de choque no pé, fazendo com que parte da força de reação do solo seja transferida para as articulações mais proximais, entre elas, o joelho, resultando em uma sobrecarga na articulação patelofemoral, o que poderia acarretar dor patelofemoral.

Apesar de existirem estudos na literatura sobre distribuição de pressão plantar, até o momento, somente quatro foram realizados em sujeitos com SDPF. Thijs et al., ${ }^{1,8}$ avaliaram a pressão plantar a fim de determinar os fatores de risco para o desenvolvimento da SDPF em militares e corredores, respectivamente. Já Aliberti et al., ${ }^{7,9}$ analisaram a distribuição da pressão plantar em sujeitos com SDPF durante a atividade de descer escadas e na marcha, respectivamente. Porém, os resultados encontrados por estes autores diferem em relação aos padrões de distribuição da pressão plantar apresentada pelos sujeitos, o que pode ter ocorrido em função de os estudos terem sido realizados com diferentes populações, instrumentos, e em situações diversas. Além disso, até a presente data não foram encontrados estudos avaliando a distribuição de pressão plantar e a cinemática do retropé em sujeitos com SDPF concomitantemente.

Diante do exposto, este estudo teve como objetivo comparar a distribuição da pressão plantar e a cinemática do retropé durante a fase de apoio da marcha de sujeitos com e sem SDPF.

\section{Métodos}

Participaram do estudo 57 sujeitos, que foram divididos em 2 grupos: o grupo SDPF (GSDPF), composto por 26 sujeitos com $23 \pm 6$ anos, $59,8 \pm 8,1 \mathrm{~kg}$ e $1,65 \pm 0,07 \mathrm{~m}$ de estatura; e o grupo controle (GC), composto por 31 sujeitos clinicamente saudáveis, com $21 \pm 4$ anos, $59,1 \pm 8,1 \mathrm{~kg}$, e $1,64 \pm 0,05 \mathrm{~m}$ de estatura. Os grupos foram pareados em relação à idade $(p=0,308)$, massa $(p=0,724)$ e estatura $(p=0,519)$. Todos os 
participantes assinaram o termo de consentimento livre e esclarecido, o qual foi aprovado pelo Comitê de Ética em Pesquisa local (protocolo ${ }^{\circ} 33 / 2010$ )

Como critérios de inclusão no GSDPF, foram considerados sujeitos que apresentaram dor anterior ou retropatelar, exacerbada por pelo menos três das seguintes situações: subir ou descer escadas, agachar por tempo prolongado, ajoelhar, correr, permanecer sentado por longos períodos, e ao praticar esportes; início insidioso dos sintomas sem relação com um evento traumático; dor igual ou maior do que $2 \mathrm{~cm}$ na escala visual numérica (EVN, que vai de $0 \mathrm{~cm}$ a $10 \mathrm{~cm}$ ) na articulação patelofemoral nos 7 dias que precederam o teste; e dor, de qualquer grandeza, em 2 testes funcionais com duração de 30 segundos cada um (agachar a 90 graus e descer um degrau com $25 \mathrm{~cm}$ de altura). ${ }^{10}$

Os critérios de inclusão para o GC foram: ausência de histórico de lesão meniscal ou ligamentar, trauma, cirurgia ou fratura do membro inferior; ausência de histórico de dor na articulação do joelho ou na articulação patelofemoral (dor de $0 \mathrm{~cm}$ na EVN); ${ }^{11}$ ausência de qualquer problema nas articulações do quadril e do pé, doença neurológica ou do sistema osteomioarticular; não ter realizado tratamento fisioterápico no membro inferior; e ausência dor, de qualquer grandeza, durante a realização dos testes funcionais com duração de 30 segundos cada um (agachar a 90 graus; descer um degrau com $25 \mathrm{~cm}$ de altura). ${ }^{10}$

Os critérios de exclusão, para ambos grupos, foram: histórico de trauma nos membros inferiores, lesão meniscal ou ligamentar do joelho; ${ }^{10}$ luxação patelar recidiva; e histórico de cirurgia no joelho ou nos membros inferiores. ${ }^{11}$

A avaliação da distribuição da pressão plantar foi realizada por meio do sistema de medição de pressão Pedar-X (Novel GmbH Inc., Munique, Alemanha), com frequência de aquisição de $100 \mathrm{~Hz}$. Todas as palmilhas foram calibradas de acordo com as especificações do fabricante. Todos os sujeitos utilizaram um calçado padrão (sapatilha Moleca, Calçados Beira Rio SA, Novo Hamburgo, RS, Brasil). As palmilhas foram colocadas dentro do calçado e conectadas a um condicionador inserido em um cinto fixo na cintura dos sujeitos. Este condicionador se comunicava e transferia os dados para o computador por Bluetooth, facilitando desta forma o deslocamento do sujeito pelo local das avaliações.

Para a avaliação da cinemática do retropé, foi utilizada uma filmadora digital (HandyCam DCR-SR65, Sony, Minato, Tóquio, Japão), a qual foi posicionada em um tripé a uma altura de $50 \mathrm{~cm}$ do solo e distância de $3 \mathrm{~m}$ do sujeito, sendo as imagens adquiridas no plano frontal posterior do sujeito com uma frequência de aquisição de $60 \mathrm{~Hz}$.

Quatro marcadores esféricos foram colocados no sujeito, nos seguintes pontos anatômicos: um marcador no centro do calcanhar, logo acima da sola da sapatilha (1), outro no centro do calcanhar, na inserção do tendão de Aquiles (2), um terceiro no centro do tendão de Aquiles, na altura do maléolo medial (3), e um quarto $15 \mathrm{~cm}$ acima do terceiro marcador, no centro da perna $(4)^{6,12,13}$ (-Figura 1 ).

Os sujeitos foram orientados a deambular por um percurso de $8 \mathrm{~m}$ em 5 tentativas. A velocidade da marcha foi monitorada,

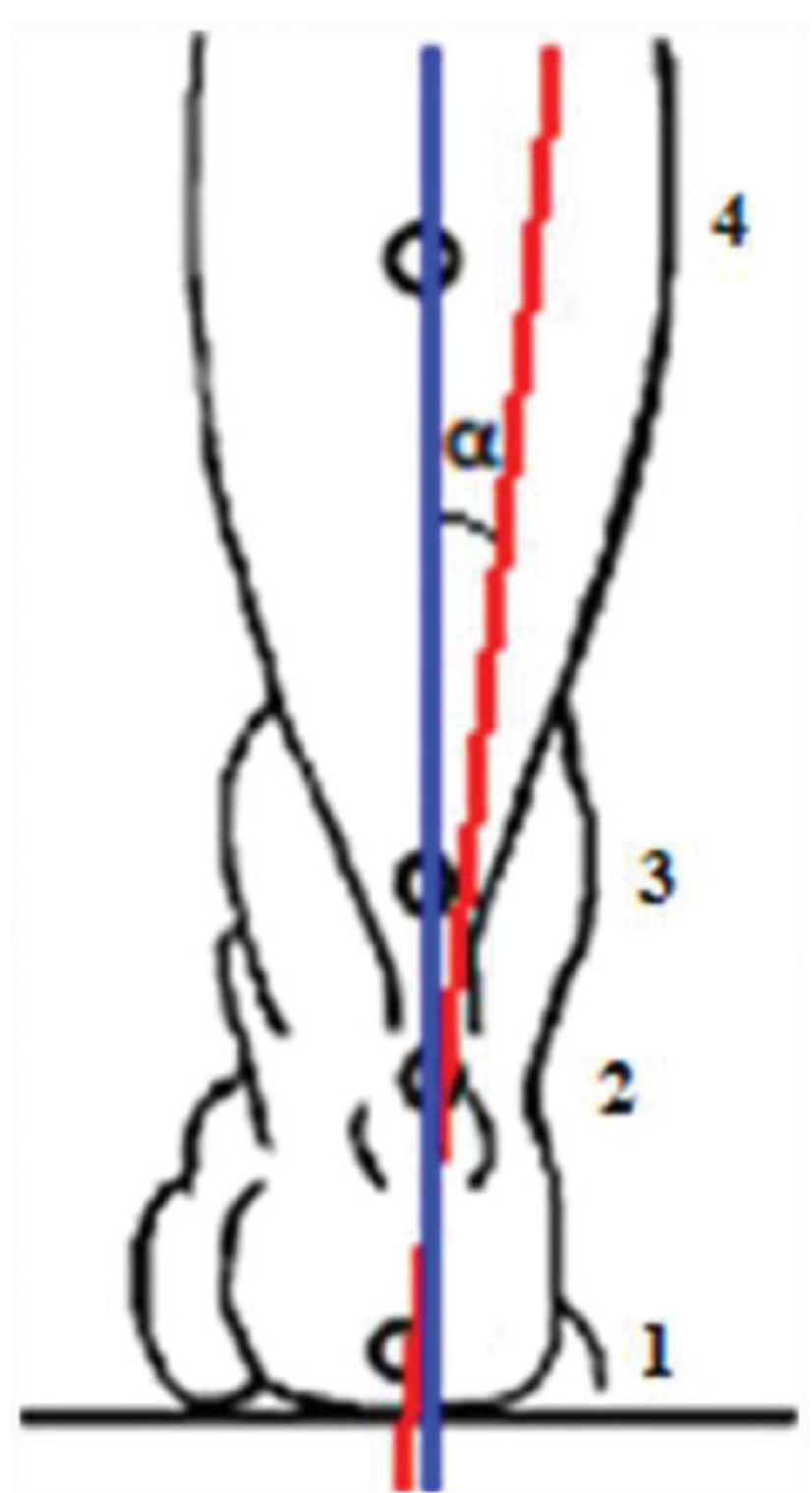

Fig. 1 Posicionamento dos marcadores para o cálculo do ângulo do retropé: 1) logo acima da sola da sapatilha; 2) centro do calcanhar, na inserção do tendão de Aquiles; 3 ) centro do tendão de Aquiles, na altura do maléolo medial; 4) $15 \mathrm{~cm}$ acima do terceiro marcador, no centro da perna.

porém não controlada. Não foram observadas diferenças entre os grupos em relação à velocidade da marcha $(p=0,7)$.

Para o cálculo das coordenadas reais, um sistema de calibração bidimensional foi colocado no plano das filmagens. Este calibrador constou de 8 pontos, de dimensões de $61 \mathrm{~cm}$ no eixo $\mathrm{x}$ e $80 \mathrm{~cm}$ no eixo $\mathrm{y}$, e um ponto fixo posicionado ao lado do calibrador.

\section{Tratamento dos dados}

\section{Distribuição da pressão plantar}

Para a análise dos dados referentes à distribuição da pressão plantar, foram descartados 1,5 metro inicial e final da caminhada e o primeiro e último passo, a fim de evitar o efeito da aceleração e desaceleração do movimento. Foram analisados, em média, 10 passos por sujeito. 


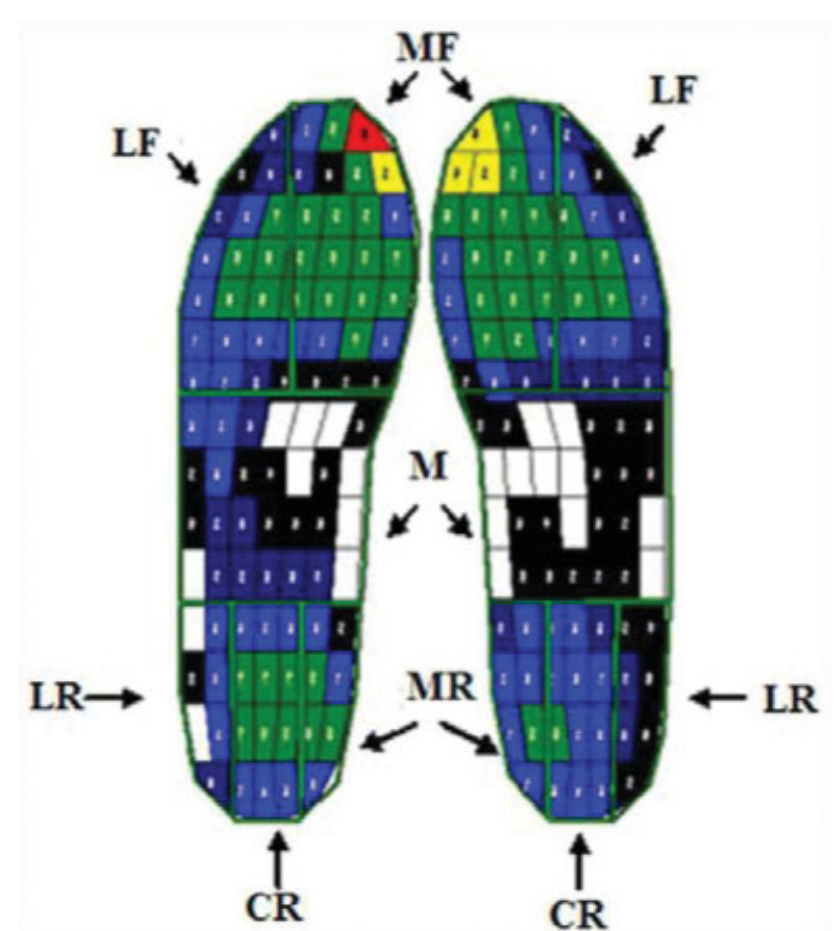

Fig. 2 Pé dividido em seis regiões, conforme a máscara aplicada. Abreviaturas: AL, antepé lateral; AM, antepé medial; M, médiopé; RC, retropé central; RL, retropé lateral; RM, retropé medial.

A superfície plantar foi dividida em retropé medial, retropé central, retropé lateral, antepé medial, antepé lateral, ${ }^{7}$ e médio pé (-Figura 2).

Foi analisado o pico de pressão (KPa) nas 6 áreas plantares que se ajustaram via software proporcionalmente à largura e ao comprimento do pé de cada sujeito.

\section{Cinemática}

Para a análise cinemática do retropé, foram analisados cinco passos. ${ }^{14-16}$ Para estimar a pronação do pé durante a marcha, foi utilizado o ângulo de eversão do retropé. ${ }^{6,17,18}$ A digitalização das imagens foi realizada no Ariel Performance Analysis System (APAS, Ariel Dynamics Inc., Trabuco Canyon, CA, EUA), e os dados foram filtrados digitalmente com frequência de corte de $6 \mathrm{~Hz}$. Foi analisado o valor máximo do ângulo de eversão do retropé durante a fase de apoio da marcha e a porcentagem da fase de apoio em que o ângulo foi atingido. Este ângulo foi definido pela interseção das linhas que formam o segmento perna com o segmento pé ( - Figura 1). A eversão foi considerada positiva, e a inversão, negativa. A fase de apoio da marcha foi considerada a partir do instante do toque do calcanhar ao solo até o desprendimento dos dedos.

Os dados cinemáticos foram normalizados na base do tempo, ajustada de $0 \%$ a $100 \%$ para a fase de apoio da marcha, com intervalos de $1 \%$, usando como referência o instante do toque do calcanhar ( $0 \%)$ até o desprendimento dos dedos do solo (100\%). A normalização foi realizada em rotina no programa Matrix Laboratory (MATLAB, MathWorks, Natick, MA, EUA). Foram analisados os dados do membro com dor na articulação patelofemoral do GSDPF e, em casos de disfunção bilateral, foi considerado o membro com maior intensidade de dor. No GC, foram analisados os dados do membro dominante, o qual foi determinado pelo membro que os sujeitos utilizavam para chutar uma bola. ${ }^{19}$

\section{Análise estatística}

Para a análise estatística, foi utilizado o programa Statistical Package for the Social Sciences (SPSS, SPSS Inc., Chicago, IL, EUA), versão 17.0, e a estatística descritiva foi usada para a caracterização dos sujeitos. O teste de Shapiro-Wilk evidenciou dados de pressão plantar e cinemáticos com distribuição gaussiana. Foi utilizado teste $t$ independente para testar a homogeneidade dos sujeitos (idade, massa, altura e velocidade da marcha), comparar o valor máximo do ângulo do retropé durante a fase de apoio da marcha em superfície plana, a porcentagem da fase de apoio da marcha em que este ângulo foi atingido pelo GSDPF e GC, e a porcentagem da fase de apoio da marcha que cada grupo permaneceu com o pé em eversão. $A$ análise de variância (analysis of variance, ANOVA) $2 \times 6$ ( 2 grupos $x 6$ regiões plantares, sendo as 6 regiões plantares consideradas medidas repetidas) foi utilizada para comparar o pico de pressão (Kpa) nas 6 regiões plantares entre o GSDPF e GC.O nível de significância adotado foi de $p \leq 0,05$.

\section{Resultados}

Não foi observado efeito de grupo $(\mathrm{F}=0,30 ; p=0,58)$, nem interação entre grupo e regiões plantares $(\mathrm{F}=0,66 ; p=0,65)$ no pico de pressão $(\mathrm{KPa})$ durante a marcha em superfície plana (-Figura 3).

Não foram constatadas diferenças significativas entre sujeitos com e sem SDFP na magnitude do ângulo máximo de eversão do retropé durante a fase de apoio da marcha em superfície plana. No entanto, o GSDPF atingiu o ângulo máximo de eversão do retropé mais cedo $(p=0,01)$ dentro da fase de apoio (32,56 $\pm 22,11 \%$ ) em comparação ao GC $(46,14 \pm 18,02 \%)$, e, consequentemente, permaneceu uma menor porcentagem da fase de apoio da marcha com o pé em eversão $(p=0,04)$ (GSDPF: $33,90 \pm 21,76 \%$; GC: 46,48 $\pm 18,21$ ) (-Tabela 1).

\section{Discussão}

A avaliação da distribuição da pressão plantar não evidenciou diferenças entre os grupos em relação ao pico de pressão nas seis regiões plantares analisadas durante a fase de apoio da marcha em superfície plana. Aliberti et al. ${ }^{7}$ analisaram a distribuição da pressão plantar durante a fase de apoio ao descer escadas em sujeitos com SDPF. No entanto, estes autores observaram um contato medialmente direcionado no retropé e médio pé, assim como menores cargas plantares durante o movimento de descer escadas nos sujeitos com SDPF. Os menores picos de pressão nestes sujeitos ao descer escadas foram relacionados a uma tentativa de redução da força de reação na articulação patelofemoral, objetivando diminuir a sobrecarga e, consequentemente, a dor.

Em outro estudo, Aliberti et al. ${ }^{9}$ avaliaram a distribuição da pressão plantar em três subfases do apoio da marcha (contato inicial, apoio médio e propulsão) em sujeitos com SDPF, e observaram um contato inicial medialmente direcionado no 


\section{Marcha em superfície plana}

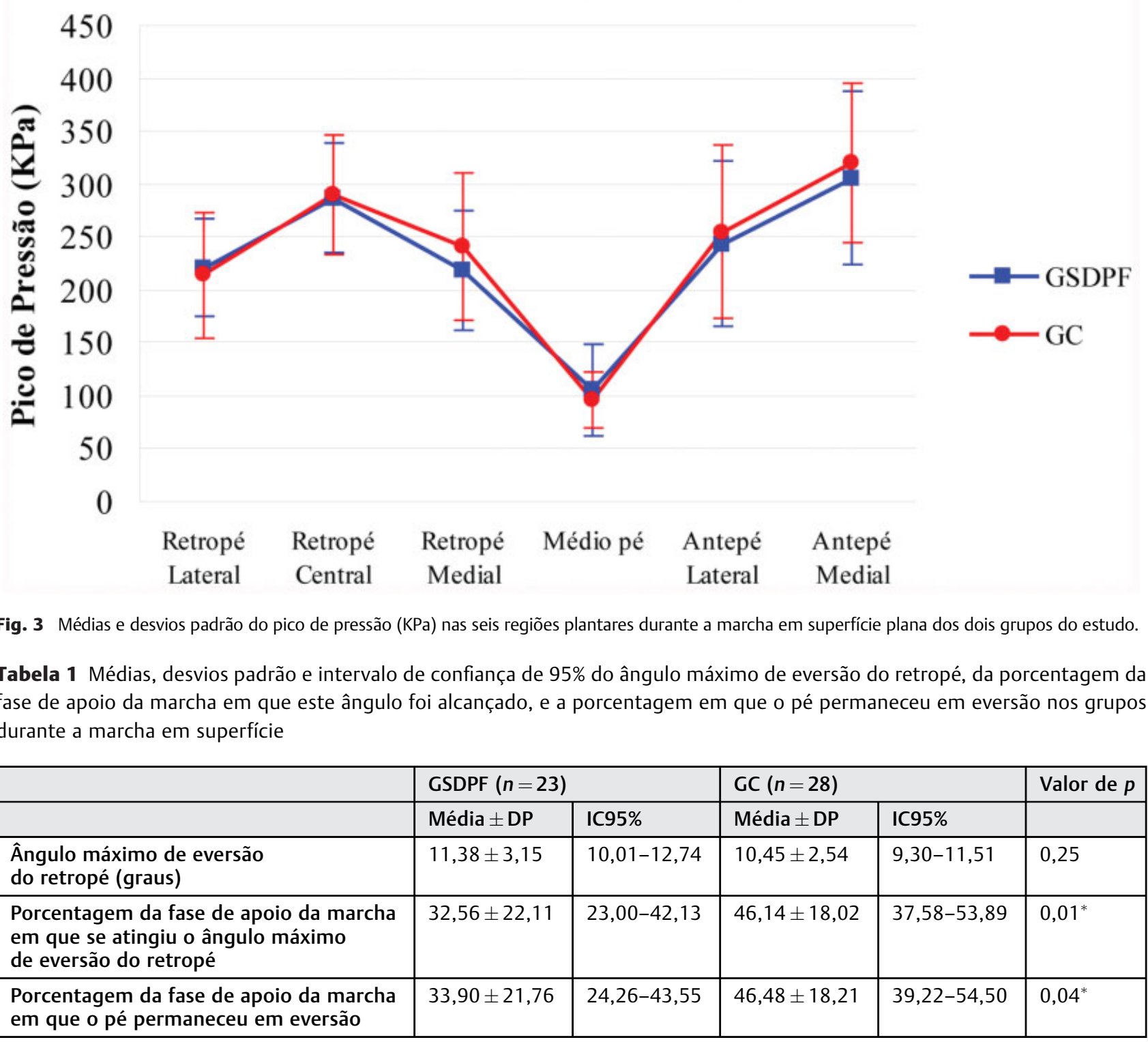

Abreviaturas: DP, desvio padrão; IC95\%, intervalo de confiança de 95\%; GC, grupo controle; GSDPF, grupo da síndrome da dor patelofemoral. Nota: *Diferença estatisticamente significativa.

retropé, e uma propulsão mais lateralizada no antepé. Segundo eles, a entrada mais evertida do pé no contato inicial parece ter reduzido a excursão da pronação inicial, que deve ocorrer nesta fase para a absorção da carga. Consequentemente, evidenciou-se um aumento na área de contato no antepé lateral ainda no apoio médio, que culminou com um desprendimento do pé mais lateral e redução no pico de pressão no antepé medial durante a propulsão.

Algumas diferenças metodológicas entre o presente estudo e os de Aliberti et al. ${ }^{7,9}$ poderiam explicar os achados conflitantes entre eles. Em nosso estudo, optamos por utilizar um calçado padrão durante a marcha (sapatilha Moleca), pois a maioria das atividades funcionais realizadas no dia a dia ocorre com calçados. Já nos estudos de Aliberti et al., ${ }^{7,9}$ os sujeitos utilizaram somente uma meia durante a coleta dos dados. Adicionalmente, os autores controlaram a cadência dos sujeitos e, consequentemente, a velocidade de sua marcha. Em nosso estudo, não fizemos tal controle, pois acreditamos que este poderia alterar o padrão da marcha dos sujeitos. Nos estudos de Aliberti et al.,, ${ }^{7,9}$ a amostra foi composta predominantemente por mulheres, mas houve a participação de alguns homens. Já no nosso estudo, todos os participantes eram do gênero feminino. Adicionalmente, na avaliação da marcha, Aliberti et al. ${ }^{9}$ analisaram-na nas três subfases do apoio, diferente do nosso estudo, em que a avaliamos em todo o apoio.

Thijs et al., ${ }^{1}$ ao investigar os fatores de risco intrínsecos para o desenvolvimento da SDPF em mulheres, constataram a presença de três fatores de risco relativos à marcha que poderiam predispor o desenvolvimento da SDPF: distribuição de pressão mais lateralizada no contato inicial do pé, redução no tempo de pressão máxima no quarto metatarso, e um atraso 
na mudança lateromedial do centro de pressão (CP) no contato do antepé durante a marcha. Segundo os autores, estas alterações podem causar uma redução na absorção de choque no pé, fazendo com que a maior parte das forças de reação do solo sejam transferidas para as articulações mais proximais, entre elas, o joelho, resultando em uma sobrecarga na articulação patelofemoral e, consequentemente, na dor patelofemoral. No entanto, estes achados, embora relevantes, não podem ser generalizados para toda a população com SDPF, nem comparados diretamente com o presente estudo, uma vez que foram realizados em uma população específica (militares), e com um instrumento diferente (plataforma de pressão FootScan).

Segundo Santos, ${ }^{6}$ em estudo feito com sujeitos normais e com aumento no ângulo de eversão do retropé, existe uma relação direta positiva entre o ângulo máximo de eversão do retropé e a distribuição da pressão plantar nas regiões do retropé (porções medial e lateral)e do médio pé (porção medial). No grupo sem e naquele com eversão excessiva, o padrão de distribuição da pressão plantar e da área de contato nas regiões plantares foram semelhantes, embora sendo sempre maior nos sujeitos com eversão excessiva.

Hipotetizamos que os sujeitos com SDPF apresentariam uma maior eversão do retropé na marcha em superfície plana em relação aos sujeitos do GC, e que esta poderia levar a alterações na distribuição da pressão plantar, com maiores valores para o pico de pressão nas regiões mediais do pé. No entanto, esta hipótese não se confirmou ao longo do estudo, uma vez que, por meio da análise cinemática do retropé, pôde-se constatar que ambos os grupos apresentaram eversão excessiva do retropé (GSDPF: $11,38^{\circ}+/-3,15^{\circ}$; GC: $\left.10,45^{\circ}+/-2,54^{\circ}\right)$, segundo as classificações de Cheung e $\mathrm{Ng},{ }^{12}$ e Santos. ${ }^{6}$ Assim, acreditamos que a presença desta eversão excessiva tanto no GSDPF quanto no GC poderia explicar a similaridade na distribuição da pressão plantar entre os grupos. O ângulo máximo de eversão do retropé durante a fase de apoio da marcha em superfície plana não evidenciou diferença entre o GSDPF e o GC, corroborando os achados de Levinger e Gilleard. ${ }^{17}$ No entanto, não existe consenso na literatura em relação aos valores angulares considerados normais para o movimento de eversão do retropé. ${ }^{20}$ Segundo Cheung e $\mathrm{Ng},{ }^{12}$ um ângulo de eversão do retropé acima de $6^{\circ}$ pode ser considerado excessivo. Já Santos ${ }^{6}$ classifica um ângulo máximo de eversão do retropé igual ou maior do que $8^{\circ}$ como eversão excessiva, e Cornwall e McPoil, ${ }^{21}$ maior do que $10^{\circ}$. Os valores encontrados por Santos ${ }^{6}$ para os sujeitos com eversão excessiva foram semelhantes $\left(10,7^{\circ}\right)$ aos encontrados em ambos os grupos do nosso estudo, sugerindo que nossos sujeitos poderiam apresentar pés com eversão excessiva.

No presente estudo, verificou-se que o aumento no ângulo de eversão do retropé não esteve relacionado com os sintomas patelofemorais, uma vez que os sujeitos assintomáticos também apresentaram maiores valores para este ângulo, indicando que uma postura do pé mais evertida pode não estar relacionada com a SDPF, confirmando os achados de alguns autores. ${ }^{17,18,22}$ Além disso, Thijs et al. ${ }^{1}$ afirmam que se deve ter cuidado ao atribuir a causa dos sintomas patelofemorais a um aumento na eversão do retropé.
No entanto, esta eversão pode ser considerada anormal não só se for excessiva em relação ao valor angular normal necessário para a locomoção, como também se ocorrer em um padrão temporal inadequado ${ }^{2}$ dentro do ciclo da marcha. Um tempo anormal de eversão pode interromper a sequência temporal de movimentos articulares da extremidade inferior. ${ }^{17}$ No presente estudo, o GSDPF atingiu o ângulo máximo de eversão do retropé mais cedo dentro da fase de apoio da marcha em relação ao GC, evidenciando que os sujeitos com SDPF realizaram uma eversão mais rápida do retropé após o contato do calcanhar com o solo do que os sujeitos do GC. Consequentemente, foi constatado que os sujeitos com SDPF permaneceram menos tempo com o pé em eversão na fase de apoio da marcha dos que os sujeitos do GC.

Moseley et al. ${ }^{20}$ ao analisar a cinemática tridimensional do retropé durante a fase de apoio da marcha de 14 homens saudáveis, constataram uma eversão gradual do retropé a partir do contato do calcanhar chegando a um máximo de $7,3^{\circ}$ a aproximadamente $57 \%$ da fase de apoio, previamente à elevação do calcanhar. No nosso estudo, os sujeitos com SDPF alcançaram o ângulo máximo de eversão do retropé a 32,56\%, e os sujeitos do GC, a 46,14\% da fase de apoio da marcha. Outros estudos também encontraram a eversão máxima ocorrendo mais cedo ${ }^{18,23}$ dentro do ciclo da marcha nos sujeitos com SDPF em relação ao GC. Em contraste, outros autores ${ }^{17}$ relataram que a eversão máxima ocorreu mais tarde nos sujeitos com SDFP quando comparados a sujeitos sem a síndrome.

Barton et al. ${ }^{18}$ também observaram, em sujeitos com SDPF, um pico de eversão do retropé mais cedo no ciclo da marcha em comparação com sujeitos sem esta afecção (32,7\% versus 36,5\%, respectivamente). No entanto, os autores apontam a existência de uma grande variabilidade quanto ao tempo para atingir o pico deste ângulo entre os indivíduos, atribuindo esta variabilidade aos achados inconsistentes entre os estudos. Sendo assim, eles sugerem a existência de subpopulações de indivíduos com SDPF com diferentes padrões de comportamento. Nossos achados também apontaram a existência desta variabilidade (intervalo de confiança de 95\% [IC95\%]: 23,00\%-42,13\%), o que poderia também sugerir a existência destas subpopulações na SDFP. No entanto, deve-se ter cautela quanto a esta afirmação, pois esta variabilidade foi observada também nos sujeitos do GC no presente estudo (IC95\%: 37,58\%-53,89\%).

Segundo Barton et al. ${ }^{18,23}$ o pico de eversão do retropé mais cedo no GSDPF pode indicar uma eversão mais rápida após o contato do calcanhar e maiores e mais rápidas cargas no joelho e articulação patelofemoral. A modificação no padrão temporal deste movimento poderia originar assincronia entre flexão do joelho, eversão e rotação tibial interna. $O$ joelho seguiria em extensão normalmente, mas o pé e, consequentemente, a tíbia não reverteriam suas ações. Esta situação pode gerar desencontro entre os movimentos de fêmur e tíbia que geraria excessivas forças de estresse e deformação no joelho. Alternativamente, isso pode resultar em compensação no quadril e no fêmur, aumentando potencialmente o risco de lesões, como a SDPF, especialmente durante a corrida. ${ }^{24}$ Este pico de eversão mais cedo no GSDPF pode ter ocorrido em parte pela estrutura do pé destes sujeitos. Sendo assim, esta relação pode estar 
ligada ao desenvolvimento da SDPF, indicando que uma postura do pé mais pronada resulta em uma eversão dinâmica mais rápida em pessoas que têm fatores de risco para o desenvolvimento da SDPF. No nosso estudo, pelo fato de não termos avaliado o tipo de pé dos sujeitos, não podemos afirmar se a ocorrência do pico de eversão mais cedo tem ou não relação com os tipos de pés (normal, pronado ou supinado).

Por outro lado, Levinger e Gilleard ${ }^{17}$ observaram, nos sujeitos com SDPF, pico de eversão do retropé mais tarde na fase de apoio da marcha comparado ao GC (39\% no GC e 46\% no GSDPF). $\mathrm{O}$ atraso no pico de eversão pode ter sido uma tentativa de atenuar o choque durante o início do apoio. No entanto, os autores não deixam claro em seu estudo se a alteração no movimento do retropé no GSDPF reflete uma modificação na marcha para evitar a dor ou um fator de causa inerente.

Sugere-se a realização de estudos futuros que avaliem a cinemática durante atividades com maior demanda funcional, como subir e descer escadas e rampas, avaliando simultaneamente a cinemática do retropé e do joelho, a fim de verificar se realmente existe relação entre estes movimentos e o desenvolvimento ou agravo da SDPF. Além disso, pesquisas futuras avaliando a velocidade do movimento de eversão do retropé e seu impacto nas cargas do joelho durante a marcha em indivíduos com SDPF são necessárias.

\section{Conclusão}

Sujeitos com SDPF evidenciaram ângulo máximo de eversão mais cedo dentro da fase de apoio da marcha do que os sujeitos do GC, o que poderia levar a maiores e mais rápidas cargas na articulação patelofemoral e ao desenvolvimento ou agravo da SDPF. Este achado pode ser particularmente importante quando consideramos estratégias de tratamento ou prevenção para a SDFP. Teoricamente, as estratégias de tratamento objetivam a redução da eversão do retropé, mas o conhecimento das mudanças temporais e sua correção pode ter efeitos globais similares na mobilidade do membro inferior e, portanto, desfecho clínico otimizado.

Conflitos de Interesse

Os autores declaram não haver conflito de interesses.

\section{Agradecimentos}

Aos fisioterapeutas Marlon Francys Vidmar e Luiz Fernando Bortoluzzi de Oliveira, e ao professor do curso de Fisioterapia da Universidade de Passo Fundo (UPF), Gilnei Lopes Pimentel, pelo auxílio na coleta dos dados. Aos médicos César Antônio de Quadros Martins, André Kuhn, Osmar Valadão Lopes Junior, José Saggin e Paulo Renato Saggin, pelo encaminhamento das pacientes para a realização da pesquisa.

\section{Referências}

1 Thijs Y, Van Tiggelen D, Roosen P, De Clercq D, Witvrouw E. A prospective study on gait-related intrinsic risk factors for patellofemoral pain. Clin J Sport Med 2007;17(06):437-445

2 Powers CM, Maffucci R, Hampton S. Rearfoot posture in subjects with patellofemoral pain. J Orthop Sports Phys Ther 1995;22(04):155-160
3 Levinger P, Gilleard W. An evaluation of the rearfoot posture in individuals with patellofemoral pain syndrome. J Sports Sci Med 2004;3(YISI 1):8-14

4 Barton CJ, Levinger P, Menz HB, Webster KE. Kinematic gait characteristics associated with patellofemoral pain syndrome: a systematic review. Gait Posture 2009;30(04):405-416

5 Tiberio D. The effect of excessive subtalar joint pronation on patellofemoral mechanics: a theoretical model. J Orthop Sports Phys Ther 1987;9(04):160-165

6 Santos JO. Aspectos cinemáticos e cinéticos do movimento de eversão do calcanhar durante a marcha [dissertação]. Florianópolis: Universidade do Estado de Santa Catarina; 2008

7 Aliberti S, Costa MS, Passaro AC, Arnone AC, Sacco IC. Medial contact and smaller plantar loads characterize individuals with Patellofemoral Pain Syndrome during stair descent. Phys Ther Sport 2010;11(01):30-34

8 Thijs Y, De Clercq D, Roosen P, Witvrouw E. Gait-related intrinsic risk factors for patellofemoral pain in novice recreational runners. Br J Sports Med 2008;42(06):466-471

9 Aliberti S, Costa MS, Passaro AC, Arnone AC, Hirata R, Sacco IC. Influence of patellofemoral pain syndrome on plantar pressure in the foot rollover process during gait. Clinics (São Paulo) 2011;66 (03):367-372

10 Cowan SM, Bennell KL, Hodges PW. Therapeutic patellar taping changes the timing of vasti muscle activation in people with patellofemoral pain syndrome. Clin J Sport Med 2002;12(06): 339-347

11 Powers CM. Patellar kinematics, part I: the influence of vastus muscle activity in subjects with and without patellofemoral pain. Phys Ther 2000;80(10):956-964

12 Cheung RT, Ng GY. Efficacy of motion control shoes for reducing excessive rearfoot motion in fatigued runners. Phys Ther Sport 2007;8:75-81

13 Perry SD, Lafortune MA. Influences of inversion/eversion of the foot upon impact loading during locomotion. Clin Biomech (Bristol, Avon) 1995;10(05):253-257

14 Cornwall MW, McPoil TG. Comparison of 2-dimensional and 3dimensional rearfoot motion during walking. Clin Biomech (Bristol, Avon) 1995;10(01):36-40

15 Chuter VH. Relationships between foot type and dynamic rearfoot frontal plane motion. J Foot Ankle Res 2010;3(09):9

16 Diss CE. The reliability of kinetic and kinematic variables used to analyse normal running gait. Gait Posture 2001;14(02):98-103

17 Levinger P, Gilleard W. Tibia and rearfoot motion and ground reaction forces in subjects with patellofemoral pain syndrome during walking. Gait Posture 2007;25(01):2-8

18 Barton CJ, Levinger P, Webster KE, Menz HB. Walking kinematics in individuals with patellofemoral pain syndrome: a case-control study. Gait Posture 2011;33(02):286-291

19 Santos GM, Ries LGK, Sperandio FF, Say KG, Pulzatto F, Pedro VM. Tempo de início da atividade elétrica dos estabilizadores patelares na marcha em sujeitos com e sem síndrome de dor femoropatelar. Fisioter Mov 2011;24(01):125-132

20 Moseley L, Smith R, Hunt A, Gant R. Three-dimensional kinematics of the rearfoot during the stance phase of walking in normal young adult males. Clin Biomech (Bristol, Avon) 1996;11(01): 39-45

21 Cornwall MW, McPoil TG. Influence of rearfoot postural alignment on rearfoot motion during walking. Foot 2004;14: 133-138

22 Duffey MJ, Martin DF, Cannon DW, Craven T, Messier SP. Etiologic factors associated with anterior knee pain in distance runners. Med Sci Sports Exerc 2000;32(11):1825-1832

23 Barton CJ, Levinger P, Crossley KM, Webster KE, Menz HB. Relationships between the Foot Posture Index and foot kinematics during gait in individuals with and without patellofemoral pain syndrome. J Foot Ankle Res 2011;4(10):10 